\title{
Diez instrumentos para mejorar el tratamiento informativo de los jóvenes. Una experiencia de proceso participativo ${ }^{1}$
}

\section{Ten instruments to improve the coverage of youth people. A participatory experience}

\author{
Mònica Figueras Maz. Universitat Pompeu Fabra \\ Marcel Mauri de los Ríos. Universitat Pompeu Fabra \\ Resumen:
}

Los mecanismos para un tratamiento adecuado de las personas jóvenes en los medios informativos son el resultado de un proceso participativo en el que intervinieron más de 50 profesionales de la comunicación (periodistas y directivos) y agentes de juventud (concejales y técnicos de juventud) impulsado por la Secretaría de Juventud de la Generalitat de Cataluña. El objetivo ha sido implicar a ambos colectivos para que asumieran como propios los instrumentos propuestos. La experiencia se ha basado en diversas técnicas participativas articuladas a partir de un Grupo de Trabajo.

Palabras clave:

Ética periodística, juventud, periodismo, participación, autorregulación

Abstract:

In this text we propose ten instruments for an appropriate treatment of young people in the media. These mechanisms are the result of a participatory process with journalist and youth experts conducted between 2008 and 2010 and led by Secretary of Youth of the Catalan Government. The main objective of this process has been involving both groups (journalists \& youth experts) in order to adopt the proposed instruments. This experience has been focused on various participatory techniques with the participation of over 50 experts.

Keywords:

Journalism ethics; youth; journalism; participation; self-regulation

1 Investigación realizada por el Grup de Recerca en Periodisme, Universitat Pompeu Fabra. Proyecto subvencionado: “El tractament informatiu de les persones joves als mitjans de comunicación”, 2010, Agència Catalana de la Joventut (Generalitat de Catalunya). 


\section{Introducción}

Los medios de comunicación tienen una función social primordial en una sociedad democrática como es garantizar el derecho a la información de todos los ciudadanos. Sin embargo, el ejercicio de este derecho colectivo a menudo colisiona con otros intereses ya sean individuales o de grupo. En las últimas décadas, y de forma paradójica, se ha ido consolidando un periodismo que ha hecho de la información espectáculo y sensacionalismo, a la vez que se ha desarrollado una conciencia profesional responsable que apela a no publicar a cualquier precio sino que vela por las consecuencias que puede comportar. Este periodismo basado en el rigor y la responsabilidad ha ido prestando atención a las demandas de protección y defensa de diversos colectivos de los llamados "socialmente desfavorecidos" (mujeres, personas homosexuales, víctimas del terrorismo, etc.). También las personas jóvenes pueden ser consideradas como uno de estos grupos atendiendo a las desigualdades de acceso y tratamiento estereotipado en los medios de comunicación.

Según el catedrático de Antropología especialista en jóvenes Carles Feixa (1998), para que exista la juventud deben existir, por un lado, las condiciones sociales, es decir, las normas, los comportamientos y las instituciones que distinguen a los jóvenes de otros grupos de edad; y, por el otro, una serie de imágenes culturales, es decir, valores, atributos y ritos asociados específicamente a los jóvenes.

En este sentido, los medios de comunicación tienen mucho que decir. El poder socializador de la prensa, la radio, la televisión y ahora Internet es evidente y más si se tiene en cuenta que está a su alcance crear y/o reproducir estereotipos o bien intentar romperlos. Desde diversas instancias juveniles, como los organismos públicos y gobiernos, pero también desde la universidad (Giró, 2003; Alcoceda, 2007; Luzón et al., 2009; Figueras y Mauri, 2010, etc.) se ha ido alertando de que el tratamiento informativo de las personas jóvenes gravita en torno a las noticias negativas, del moralismo y del sensacionalismo. A menudo se les configura una identidad fija o unos rasgos diferenciales propios, a veces totalmente opuestos: la juventud es idealista y a la vez materialista; dinámica y al mismo tiempo pasiva, etc. La juventud representada en los medios tiene asociadas dos grandes imágenes contrapuestas: la idealizada, a menudo a la publicidad (la juventud como valor, ya sea desde el punto de vista estético, de salud o de estilo de vida) o la estigmatizada, a menudo los espacios informativos (personas egoístas, violentas, "sin valores"...).

Desde la deontología y la ética periodística existe una conciencia crítica y una reflexión profesional sobre la necesidad de hacer una información responsable que se ajuste a los valores tradicionales del periodismo y que no colisione con los derechos individuales y colectivos. En una propuesta de taxonomía de estos principios periodísticos propuesta por Alsius (1998) se pueden clasificar los valores deontológicos en cuatro grupos: verdad, libertad, responsabilidad y justicia. Cada uno de estos cuatro principios agrupa en su interior un conjunto de normas, criterios y recomendaciones que recogen los códigos deontológicos nacionales, estatales e internacionales. 
Cuando hablamos del tratamiento informativo de los grupos "socialmente desfavorecidos" hay que buscar en la categoría de justicia las recomendaciones para una cobertura lo más adecuada posible. Este principio consiste en favorecer que aquellos grupos sociales que parten con desventaja puedan recibir desde los medios un trato positivo o adecuado que contribuya a modificar o transformar la percepción social que de ellos se tiene.

Si bien es cierto que son muchas las instituciones responsables de la creación de estereotipos, el periodismo también tiene su parte de influencia en la construcción y reproducción de roles, tal y como ya indicaba Walter Lippmann en 1922. La espectacularización y simplificación de la información que se lleva a cabo desde diversos medios de comunicación añadida a la personificación e invasión de la esfera privada que hacen algunos periodistas, contribuye aún más a la creación de estereotipos. A esto hay que sumar un afán, a menudo poco reflexivo, de dotar de contenido diferencial el "quién" de la información: indicar la nacionalidad, la etnia o la condición sexual pero también la edad del protagonista de una noticia, muchas veces no aporta información relevante y, en cambio, puede conllevar el peligro de la estereotipación, sobre todo si la noticia tiene connotaciones negativas. Un enfoque periodístico que remarque excesivamente las diferencias y no ponga el acento en las semejanzas, siempre mayoritarias, puede acabar comportando incomprensión entre grupos y contribuir a la discriminación y exclusión social.

Por todo ello, es frecuente encontrar en códigos nacionales, internacionales e incluso en normas deontológicas de los propios medios referencias directas para dispensar un tratamiento justo a colectivos como las mujeres víctimas de violencia machista o de atentados terroristas. En otros casos, como los homosexuales o las personas inmigradas, las referencias en los códigos éticos no son tan abundantes ni explícitas por lo que desde los propios colectivos se ha optado por impulsar recomendaciones dirigidas a los profesionales de la información. En cuanto a jóvenes, las referencias a cómo se debe elaborar información sobre ellos son casi inexistentes, con el agravante de que son también muy pocos los documentos nacionales e incluso internacionales así como son escasas las propias recomendaciones impulsadas desde instituciones juveniles.

\section{Objetivo y metodología de trabajo}

El objetivo de la experiencia que aquí se presenta era elaborar una propuesta de mecanismos para un trato adecuado de las personas jóvenes en los medios informativos. Como objetivo específico se planteó la necesidad de implicar a todos los agentes relacionados y por ello se contó con la colaboración de más de 50 personas del ámbito de la comunicación y de la juventud en un amplio proceso participativo que se desarrolló en distintas fases entre 2008 y $2010^{2}$. Esta implicación pre-

Para elegir los participantes del proceso se tuvo en cuenta su edad. Se consideró que no se podía hablar de personas jóvenes sin darles la palabra. Por este motivo, tanto en el ámbito del periodismo como en el de los agentes de juventud, se trató que hubiera representantes de este colectivo. 
tendía conseguir que el resultado final de la elaboración de los instrumentos y mecanismos de mejora fueran asumidos como propios por todos los participantes en este proceso. Esta voluntad manifiesta de incardinación entre instituciones y profesionales de reconocido prestigio de ambos ámbitos se consideró, ya en la fase de diseño de todo el proceso, como uno de los factores clave para garantizar el éxito del proyecto.

\subsection{Grupo de trabajo}

Para implicar de forma integradora a todos los agentes que participan en la imagen de transmiten los medios sobre las personas jóvenes se creó un Grupo de Trabajo que ha tenido como principal misión hacer un seguimiento del proyecto. Los miembros que formaron este consejo, elegidos conjuntamente con la Secretaria de Joventut de la Generalitat de Catalunya (administración que financió el proyecto), representaban de forma mixta expertos en el ámbito de la juventud y en el ámbito de la comunicación.

El Grupo de Trabajo estaba constituido por miembros a título institucional y otros a título individual de reconocido prestigio en Catalunya. Los miembros institucionales fueron el Consell Nacional de la Joventut de Catalunya (con la participación de sus respectivos presidentes Agnès Rossinyol, Miquel Andreu y Víctor Albert); el Col-legi de Periodistes de Catalunya (con el miembro de la Junta, Enric Frigola); Televisió de Catalunya (con el responsable de contenidos infantiles y juveniles, Dani López); el Consell de la Informació de Catalunya (con la participación de su presidenta, Llúcia Oliva), y el Consell de l'Audiovisual de Catalunya (con la responsable del área de Estudios, Maria Corominas). Los miembros individuales fueron Salvador Alsius, director de los Estudios de Periodismo de la UPF y ex decano del Col-legi de Periodistes de Catalunya; Rosario Lacalle, catedrática de periodismo de la Universidad Autónoma de Barcelona; Vicent Partal, periodista, director de Vilaweb y responsable de desarrollo del portal Jove.Cat; Ricard Palou, periodista del diario El Punt; y Josep Maria Villena, presidente de la entidad Taula de Comunicació.

Para que el Grupo tuviera elementos para valorar y elaborar propuestas de mejora se utilizaron varios materiales:

- Los resultados de un análisis de contenido sobre la presencia de los jóvenes en los medios de comunicación líderes en Cataluña obtenidos en un estudio empírico ad hoc que realizaron previamente los mismos investigadores responsables del proceso de participación aquí descrito ${ }^{3}$.

\footnotetext{
Este estudio analizó más de 20.000 noticias publicadas por los medios líderes de audiencia en Cataluña durante el último trimestre de 2008 y los dos primeros de 2009. Los resultados de la investigación permitieron comprobar cómo los jóvenes están infrarrepresentados en los medios catalanes; cómo su presencia a menudo se relaciona con temas como la violencia; que los medios de comunicación no utilizan fuentes jóvenes para informar sobre ellos; y que la actitud del periodista tiende a ser neutral aunque en noticias donde se vinculan valores negativos, esta posición acostumbra a ser negativa hacia las personas jóvenes (Figueras, M. y Mauri, M., 2010).
} 
- $\quad$ Las conclusiones y reflexiones del grupo de discusión y entrevistas en profundidad realizados con agentes de juventud y de la comunicación para el estudio anterior.

- $\quad$ Un análisis DAFO (dificultades, amenazas, fortalezas y oportunidades) comparativo sobre buenas prácticas en actuaciones de mejora del tratamiento informativo de la juventud y otros colectivos considerados socialmente desfavorecidos a nivel estatal e internacional.

Con estos indicadores sobre la mesa, el Grupo de Trabajo se reunió en tres ocasiones. En el primer encuentro el Grupo se constituyó formalmente y se validó el diseño del proceso de trabajo. A partir de conclusiones parciales de la investigación empírica y del análisis DAFO sobre los mecanismos implementados en otros colectivos, el Grupo de Trabajo se reunió por segunda vez para empezar a plantear propuestas de mejora en el tratamiento informativo de las personas jóvenes. Entre la segunda y tercera sesión plenaria del Grupo, se realizó un seminario que consistió en un trabajo con una comisión voluntaria de miembros del Grupo de Trabajo a partir de los mecanismos de actuación analizados para ver si estas propuestas eran viables y bien recibidas. El objetivo era avanzar en propuestas para agilizar el último encuentro de trabajo en el que se debían ratificar los instrumentos elaborados. El tercer encuentro plenario sirvió para consensuar y aprobar los mecanismos de mejora del colectivo juvenil.

\subsection{Seminario de trabajo}

Con el fin de conocer las reflexiones de los agentes implicados en el día a día y confrontar visiones de los ámbitos periodístico y juvenil, se organizó un seminario de reflexión y debate en el que participaron ocho profesionales: cuatro del ámbito periodístico (directores y periodistas de medios relevantes como TV3, Catalunya Ràdio, Telecinco, ACN) y cuatro del ámbito de juventud (técnicos de juventud de ayuntamientos, representantes del Consell Nacional de la Joventut de Catalunya, técnico del Observatori Català de la Joventut).

\subsection{Talleres con profesionales}

En el marco de las VII Jornadas de Comunicación “Jóvenes y Medios de Comunicación” organizadas por la Taula de Comunicación y la Secretaría de juventud el 12 y 13 de julio de 2010, se realizaron dos talleres (que nuevamente involucraron a periodistas y agentes de juventud) para testar los instrumentos diseñados y captar la opinión de los participantes. Un taller iba dirigido a profesionales de la información y el otro a personas del ámbito de la juventud. Participaron como ponentes nuevamente periodistas y técnicos de juventud. 


\subsection{Experiencias existentes en el tratamiento informativo de diferentes colectivos "socialmente desfavorecidos"}

El análisis DAFO es una técnica que analiza la situación real de una empresa u organización con el fin de sistematizar sus Debilidades, Oportunidades, Fortalezas y Amenazas. Dado que en materia de juventud no ha habido demasiadas propuestas, se amplió el radio de acción a las actuaciones realizadas por otros colectivos, especialmente mujeres y personas inmigradas, porque se partía de la idea de que muchas de ellas podían ser extrapolables. Así, no se trataba de aplicar un análisis DAFO a un proyecto concreto, sino a muchos, de ámbito nacional o internacional, de ámbito juvenil o en otros ámbitos. Las iniciativas se agruparon en tres ámbitos diferentes de actuación:

- Ámbito de formación. Propuestas dirigidas tanto a las personas que trabajan como agentes de los colectivos afectados como a los propios periodistas para que, a través de espacios de formación, tengan un conocimiento mayor del contexto en que se enmarcan las personas que forman parte de aquel colectivo concreto. El objetivo es que un mayor conocimiento y formación reduzca la superficialidad de las informaciones y, como consecuencia, también disminuyan los posibles estereotipos.

- Ámbito de acción y de autorregulación. Códigos y recomendaciones son el instrumento más habitual que tanto desde las instituciones que trabajan en favor de estos colectivos como desde las propias corporaciones de periodistas elaboran para dar pautas a los periodistas a la hora de informar sobre noticias que tengan como protagonistas personas de estos grupos sociales.

- $\quad$ Ámbito de divulgación. Desde instituciones, entidades representantes de estos colectivos y corporaciones periodísticas se fomentan las acciones de divulgación y reconocimiento de aquellas buenas prácticas profesionales en el campo de la comunicación que fomentan una información adecuada y de calidad sobre los grupos socialmente desfavorecidos y que, a la vez, contribuyen a un periodismo más riguroso.

\section{Resultados}

El Grupo de trabajo estudió los resultados del DAFO, analizó los informes del seminario de trabajo y talleres, y finalmente aprobó diez instrumentos que por su idiosincrasia podían ser aplicables y eficaces para la realidad comunicativa y juvenil. Se indican los puntos débiles de cada uno así como los promotores que deben impulsarlos. 


\section{1. Ámbito Formativo}

Jornadas de formación y debate para periodistas

Descripción: Jornadas que tienen por objetivo incrementar el conocimiento de los profesionales de la comunicación sobre la realidad juvenil y, sobre todo, su contexto.

Destinatario: Periodistas y también agentes juveniles (miembros del tejido asociativo, investigadores, órganos reguladores, etc.).

Promotor: Organismos públicos de juventud y / o mundo asociativo juvenil conjuntamente con agentes del ámbito periodístico.

Puntos fuertes: Permite el trabajo en dos direcciones: reconocimiento y sensibilización ante la situación y adquisición de herramientas y recursos para la mejora. Facilitado el conocimiento mutuo entre agentes juventud y periodistas.

Puntos débiles: Posible falta de asistencia de periodistas. Es recomendable incidir en el trabajo a partir de temas, casos, noticias concretas y convocar a los periodistas implicados directamente, no sólo a través de una convocatoria abierta.

Encuentros de proximidad entre agentes de juventud y periodistas

Descripción: Encuentros de proximidad de carácter informal (comidas o reuniones en petit comité) en que agentes de juventud convocan a periodistas para hacer llegar posibles hechos noticiables (ya sean actos, eventos, informes,...). La finalidad es contribuir a un mayor conocimiento entre los dos sectores (juvenil y periodístico). A la larga, pueden acabar repercutiendo en un uso más elevado de fuentes informativas jóvenes y en una mejor comprensión de los contextos juveniles por parte de los periodistas.

Destinatario: Periodistas, especialmente los que tratan temas de sociedad (ámbito en el que se enmarcan la mayoría de noticias de juventud).

Promotor: Agentes de juventud de organismos públicos (Secretaría de Juventud, entidades municipales, etc.) o de la sociedad civil (entidades del Tercer Sector, etc.).

Puntos fuertes: Se trata de un instrumento habitual y de éxito en otros ámbitos, capaz de sensibilizar a los profesionales de la comunicación eficaz para dar a conocer hechos y acciones puntuales y, sobre todo, sus contextos.

Puntos débiles: Encuentro que dura poco tiempo, poca capacidad formativa. Hay que ser muy cuidadoso y riguroso en la selección del tema por el que se convoca el encuentro, un uso inadecuado o demasiado repetitivo de este mecanismo puede provocar que sea percibido como un espacio prescindible por parte de los periodistas. 


\section{- $\quad$ Guías didácticas para estudios universitarios de periodismo y de comunicación audiovisual}

Descripción: Guías didácticas que orienten al profesorado universitario sobre cómo formar a los futuros periodistas en esta materia, con el objetivo de reforzar la formación específica en juventud. Actualmente no existe ningún material que recoja los objetivos y competencias propias para un tratamiento adecuado de las personas jóvenes.

Destinatario: Profesores de periodismo, especialmente de las asignaturas de ética y deontología de la información. Promotor: Agentes de juventud (administraciones o asociaciones), universidades y agentes del ámbito del periodismo (asociaciones de periodistas, organismos reguladores...).

Puntos fuertes: Sensibilizar a los futuros profesionales sobre el tratamiento informativo de los jóvenes y facilitar el trabajo al profesorado con materiales ya elaborados de fácil aplicabilidad.

Puntos débiles: Riesgo de tener poco impacto para la facilidad de caer en el olvido del estudiante (futuro periodista). Posibilidad de hacer una acción coordinada con otros colectivos de los llamados socialmente desfavorecidos (inmigrantes, mujeres, personas con alguna discapacidad, víctimas de violencia...) para potenciar su impacto.

Guías o manuales para la comunicación de las administraciones / asociaciones en temas de juventud

Descripción: Manual o guía para la comunicación que permita a los agentes juveniles comprender los medios y saber cuáles son las claves para poder acceder. La visualización de la tarea que realizan los agentes juveniles es un elemento potencialmente noticiable. Muchas entidades e incluso las administraciones locales tienen dificultades para hacer visible su labor, la guía podría resultar útil.

Destinatario: Administraciones públicas y asociaciones o redes asociativas juveniles.

Promotor: Agentes de juventud de organismos públicos o de la sociedad civil. Puede incluir la participación de agentes del ámbito del periodismo.

Puntos fuertes: Implicar al sector que a menudo critica su invisibilidad de su acción en los medios en la reversión de esta tendencia.

Puntos débiles: Las circunstancias diferentes que estos dos tipos de agentes (instituciones juveniles públicas y asociaciones) puedan tener: para evitarlo se podrían elaborar dos documentos diferentes adaptados a cada caso. Poco impacto del manual que se puede contrarrestar con seminarios de presentación y formación o con la posible creación de una consultoría de comunicación. 


\section{2. Ámbito de acción y de autorregulación}

Recomendaciones deontológicas para medios y profesionales de la información ${ }^{4}$

Descripción: Se parte de un planteamiento de justicia compensatoria hacia un colectivo heterogéneo con desigualdad de acceso a los medios informativos. Los jóvenes hasta 18 años están protegidos por la regulación específica de los menores, a partir de esta edad no hay diferencias legales respecto a un adulto en el tratamiento informativo. Es por ello que se cree necesario un documento que oriente a los y las profesionales de la información en algunos aspectos que puedan ayudar a mejorar el tratamiento periodístico de las personas jóvenes y, en definitiva, la imagen que proyectan.

Destinatario: Periodistas.

Promotor: Organismos públicos y asociativos de juventud conjuntamente con periodistas. También es recomendable la implicación de medios de comunicación a título individual.

Puntos fuertes: Coste reducido y reconocimiento amplio si el impulso es compartido por el ámbito periodístico.

Puntos débiles: Riesgo de quedar como una lista más de recomendaciones con incidencia limitada si no se hace difusión y se consigue sensibilizar a la profesión.

\section{Observatorio de los medios}

Descripción: Organismo encargado de observar los contenidos de los medios y velar porque se cumplan determinados requisitos de la ética periodística en cuanto al tratamiento de las personas jóvenes. Este tipo de observatorio puede utilizar diferentes instrumentos, con grados de aceptación diversos por parte de los medios: informes de análisis sobre temas concretos que se hagan llegar a las redacciones para incitar a la reflexión profesional, la difusión pública de ejemplos positivos y negativos, y la divulgación y la promoción de la investigación en este campo, entre otros, son algunas de las herramientas de utilidad.

Destinatario: Periodistas, medios de comunicación, investigadores, administraciones.

4 Única propuesta implementada. La elaboración de este instrumento tuvo en cuenta las conclusiones que apuntó el estudio empírico sobre el tratamiento de los jóvenes en los medios de comunicación catalanes. También se valoraron las aportaciones y reflexiones que los profesionales de los dos ámbitos (periodismo y juventud) expusieron en los distintos foros de discusión descritos. Como resultado de este análisis cuantitativo y cualitativo, se elaboró, en el marco del proceso participativo, un decálogo que se presentó públicamente en Barcelona en julio de 2010 y fue asumido por instituciones, empresas periodísticas y agentes juveniles. El documento está accesible en la página web de la Generalitat de Catalunya, Dirección de Juventud http://premsa.gencat.cat/pres_fsvp/docs/2010/07/ 12/11/55/d63a40d6-baee-48fd-8a2a-b4dfc7a14051.pdf 
Promotor: Organismos públicos de juventud y/o mundo asociativo juvenil conjuntamente con agentes del ámbito periodístico y universidades.

Puntos fuertes: Puede ser el agente promotor responsable de algunos de los mecanismos descritos anteriormente a continuación. El trabajo permanente, específico y multidireccional puede tener más incidencia que acciones puntuales.

Puntos débiles: Viabilidad económica.

\section{3. Ámbito de divulgación}

\section{Premios y reconocimientos}

Descripción: Reconocimientos a buenas prácticas periodísticas que hayan contribuido a un tratamiento riguroso y adecuado de las personas jóvenes.

Destinatario: Periodistas y medios de comunicación.

Promotor: Organismos públicos de juventud, mundo asociativo juvenil, agentes del ámbito periodístico o algunos de ellos de forma conjunta.

Puntos fuertes: Instrumento habitual, sencillo y muy complementario con las siguientes propuestas.

Puntos débiles: La especificidad de la temática puede dificultar su éxito.

- $\quad$ Guías de recopilación de buenas prácticas de los medios

Descripción: Recopilación y edición en formato escrito o digital de ejemplos de programación respetuosa e innovadora y de buenas prácticas.

Destinatario: Periodistas, responsables de medios, profesores y estudiantes de periodismo y comunicación audiovisual.

Promotor: Organismos públicos de juventud, mundo asociativo juvenil, agentes del ámbito periodístico, universidades o algunos de estos de forma conjunta.

Puntos fuertes: Mecanismo sencillo y muy complementario con los reconocimientos (propuesta anterior) y las muestras de programación de calidad (propuesta siguiente).

Puntos débiles: Posible repercusión limitada. 


\section{- $\quad$ Muestra de programación televisiva de calidad}

Descripción: Festival de televisión centrado en aquellos productos informativos que sobresalen por la representación respetuosa de las personas jóvenes. Instrumento para difundir ideas y para facilitar la interacción entre personas del ámbito académico, periodístico (de medios locales y nacionales) y del mundo asociativo juvenil.

Destinatario: Periodistas, responsables de medios, profesores y estudiantes de periodismo y comunicación audiovisual y genéricamente toda la población.

Promotor: Organismos públicos de juventud, mundo asociativo juvenil, agentes del ámbito periodístico, universidades o algunos de estos de forma conjunta.

Puntos fuertes: Propuesta innovadora, inexistente a nivel internacional en el ámbito juvenil y complementaria a las dos anteriores. Posibilidad de dar voz a jóvenes creadores de productos comunicativos. Posibilidad de ampliar la propuesta a otros medios (radio, prensa, internet) y géneros.

Puntos débiles: Viabilidad económica.

- $\quad$ Guía de expertos o de fuentes en materia de juventud

Descripción: Guía de fuentes informativas en el ámbito de juventud que incluya, por un lado, las asociaciones juveniles (por sectores y ámbitos geográficos) y, por otro, investigadores o expertos académicos en juventud, preferentemente jóvenes. Instrumento para facilitar la labor periodística y superar el déficit de fuentes juveniles.

Destinatario: Periodistas.

Promotor: Organismos públicos de juventud y / o mundo asociativo juvenil.

Puntos fuertes: Material al alcance del periodista que permite llenar un vacío existente y facilitar su tarea para poder informar rigurosamente sobre las personas jóvenes.

Puntos débiles: La alta rotación de responsables en el mundo asociativo juvenil dificultará la vigencia del material, pero en soporte digital se facilitaría la actualización.

\section{Conclusiones}

Para la implementación de cualquiera de los mecanismos analizados será necesario que los promotores aquí sugeridos o bien otros apuesten por ellos. En todo caso, como ha ocurrido con su diseño, será imprescindible que se reúnan, como mínimo, las dos características siguientes: interacción entre los agentes implicados (que sea referencia tanto para periodistas 
como para los que trabajan con jóvenes) y responsabilidad compartida (que las propuestas se dirijan a periodistas pero también ayuden a reflexionar críticamente el trabajo de los propios agentes de juventud).

De poco sirve crear instrumentos pensados para ayudar a mejorar la presencia o la imagen de la juventud en los medios si periodistas y profesionales no comparten puntos de vista, matizan discrepancias, se explican mutuamente los contextos propios y buscan puntos de encuentro. La responsabilidad que tienen los medios de comunicación en la creación y/o propagación de estereotipos es obvia, pero también es necesaria una reflexión desde el mundo juvenil sobre qué pasos se pueden seguir para evitar contribuir a ello. Es por este motivo que el diseño de estas propuestas y mecanismos no ha ido dirigido exclusivamente a periodistas, como podía esperarse en un principio, sino también a agentes de juventud. En el mismo sentido, se considera importante la difusión de estos instrumentos en las facultades de periodismo pero también en las de formación de agentes de juventud.

De momento, no se puede evaluar el grado de implementación de estos instrumentos pero sí creemos que ya es un indicador de éxito la participación en el diseño y la asunción de los resultados por parte de personas relevantes en Cataluña en el ámbito implicadas a título individual y sobre todo institucional, como el Col-legi de Periodistes de Catalunya, el Consell de l'Audiovisual de Catalunya, el Consell Nacional de la Joventut o la Secretaria de Joventut de la Generalitat.

\section{Referencias bibliográficas}

Alcoceba, J. A. \& Cadilla, M. (dirs.) (2007): La imagen de los jóvenes en los medios de comunicación. Madrid: Instituto de la Juventud.

Alsius, S. (1998): Ètica i periodismo. Barcelona: Pòrtic.

Baca, V. (1998): Imágenes de los jóvenes en los medios de comunicación de Masas. Madrid: Instituto de la Juventud-Fundación de Ayuda a la Drogadicción.

Giró, X. (2003): “La imatge de la joventut a la premsa escrita. Valors, política i violencia”, Anàlisi, n. 30.

Feixa, C. (1998): De jóvenes, bandas y tribus. Barcelona: Ariel.

Figueras, M. y Mauri, M (2010): Mitjans de comunicación i joves. Barcelona: Generalitat de Catalunya, Secretaria de la Joventut, col-lecció Aportacions, n. 45.

Lippmann, W. (1922): Stereotypes, Public Opinion and the Press. Mcmillan Publishing.

Luzón, V. et al. (2009): La imagen de los/as adolescentes en el prime time televisivo. Transmisión, consumo y recepción. Disponible en: http://www.inmujer.migualdad.es/mujer/mujeres/estud_inves/820.pdf. [Consultado en diciembre 2011]. 